Western University

Scholarship@Western

Brain and Mind Institute Researchers'

Publications

$7-1-2018$

\title{
Longitudinal diffusion tensor imaging changes in early Parkinson's disease: ICICLE-PD study
}

Thais Minett

University of Cambridge

Li Su

University of Cambridge

Elijah Mak

University of Cambridge

Guy Williams

University of Cambridge

Michael Firbank

University of Newcastle upon Tyne, Faculty of Medical Sciences

See next page for additional authors

Follow this and additional works at: https://ir.lib.uwo.ca/brainpub

Citation of this paper:

Minett, Thais; Su, Li; Mak, Elijah; Williams, Guy; Firbank, Michael; Lawson, Rachael A.; Yarnall, Alison J.; Duncan, Gordon W.; Owen, Adrian M.; Khoo, Tien K.; Brooks, David J.; Rowe, James B.; Barker, Roger A.; Burn, David; and O’Brien, John T., "Longitudinal diffusion tensor imaging changes in early Parkinson's disease: ICICLE-PD study" (2018). Brain and Mind Institute Researchers' Publications. 964.

https://ir.lib.uwo.ca/brainpub/964 


\section{Authors}

Thais Minett, Li Su, Elijah Mak, Guy Williams, Michael Firbank, Rachael A. Lawson, Alison J. Yarnall,

Gordon W. Duncan, Adrian M. Owen, Tien K. Khoo, David J. Brooks, James B. Rowe, Roger A. Barker, David Burn, and John T. O’Brien 


\title{
Longitudinal diffusion tensor imaging changes in early Parkinson's disease: ICICLE-PD study
}

\author{
Thais Minett ${ }^{1,2}$ (D) Li Su $^{3,17} \cdot$ Elijah Mak $^{3} \cdot$ Guy Williams ${ }^{4} \cdot$ Michael Firbank $^{5} \cdot$ Rachael A. Lawson $^{5} \cdot$ Alison J. Yarnall $^{5}$. \\ Gordon W. Duncan $^{6}$. Adrian M. Owen ${ }^{7,8} \cdot$ Tien K. Khoo ${ }^{9}$. David J. Brooks ${ }^{5,10,11}$. James B. Rowe ${ }^{12,13,14}$. \\ Roger A. Barker ${ }^{15}$. David Burn ${ }^{16} \cdot$ John T. O'Brien ${ }^{3}$
}

Received: 25 January 2018 / Revised: 13 April 2018 / Accepted: 16 April 2018

c) Springer-Verlag GmbH Germany, part of Springer Nature 2018

\begin{abstract}
Objective To investigate whether white matter microstructural changes can be used as a predictor of worsening of motor features or cognitive decline in patients with Parkinson's disease and verify whether white matter microstructural longitudinal changes differ between patients with Parkinson's disease with normal cognition and those with mild cognitive impairment. Methods We enrolled 120 newly diagnosed patients with early stage Parkinson's disease (27 with mild cognitive impairment and 93 with normal cognition) along with 48 controls. Participants were part of the incidence of cognitive impairment in cohorts with longitudinal evaluation in Parkinson's disease study and were assessed at baseline and 18 months later with cognitive, motor tests and diffusion tensor imaging. The relationships between fractional anisotropy and mean diffusivity with disease status, cognitive and motor function were investigated.

Results At baseline, patients with early stage Parkinson's disease had significantly higher widespread mean diffusivity relative to controls, regardless of cognitive status. In patients with Parkinson's disease/mild cognitive impairment, higher mean diffusivity was significantly correlated with lower attention and executive function scores. At follow-up frontal mean diffusivity increased significantly when comparing patients with Parkinson's disease/mild cognitive impairment with those with normal cognition. Baseline mean diffusivity was a significant predictor of worsening of motor features in Parkinson's disease. Conclusions Mean diffusivity represents an important correlate of cognitive function and predictor of motor impairment in Parkinson's disease: DTI is potentially a useful tool in stratification of patients into clinical trials and to monitor the impact of treatment on motor function.
\end{abstract}

Keywords Parkinson's disease · Mild cognitive impairment · Assessment of cognitive disorders $\cdot$ Longitudinal study $\cdot$ DTI

\section{Introduction}

The incidence of dementia is five times greater in patients with Parkinson's disease (PD) than in the general population [1]. Whether dementia is an inevitable event in PD is yet to be established, but $83 \%$ of patients have dementia if alive at 20 years from onset [2]. Cognitive changes start early in PD with $36 \%$ of newly diagnosed patients having mild cognitive impairment (PD-MCI) [3], of whom two-third develop

Electronic supplementary material The online version of this article (https://doi.org/10.1007/s00415-018-8873-0) contains supplementary material, which is available to authorized users.

Thais Minett

thaisminett@hotmail.com

Extended author information available on the last page of the article dementia within 4 years [4]. Elucidating the pathophysiological mechanisms underlying cognitive impairment in PD may, therefore, influence patients' treatment selection and response monitoring early in their illness.

White matter lesions correlate with motor and cognitive abnormalities in otherwise healthy elderly individuals, and are predicted to contribute to clinical symptoms in PD [5]. Increased volume of white matter lesions has been described in PD, especially in those with dementia [6]. Even slight changes in the WM, as identified by diffusion tensor imaging (DTI), might contribute to the cognitive decline in PD $[7,8]$. Diffusion tensor parameters such as mean diffusivity (MD) and fractional anisotropy (FA) are established markers of white matter microstructure and can demonstrate subtle damage even when clinical structural imaging shows normal appearing white matter [7]. 
We therefore, proposed that white matter change is a major contributor to cognitive decline in PD. Although the underlying pathophysiological process of PD dementia is likely to be heterogeneous [9] we have previously shown that in the early stages of PD, there are increases in MD even when there is no significant grey matter volume loss [10], suggesting that MD may be an early biomarker of this state. Monitoring white matter changes with DTI offers a non-invasive biomarker which could reflect a mechanism underlying cognitive impairment in PD. We tested the specific hypothesis that longitudinal white matter microstructural changes, characterised by decrease in FA and increase in MD, are more pronounced among patients with PD-MCI than those with PD who are cognitively normal at baseline. Further, we tested whether baseline white matter abnormalities can be used as a predictor of cognitive decline or worsening of motor impairment.

\section{Methods}

The study was approved by the Newcastle and North Tyneside Research Ethics Committee and has, therefore, been performed in accordance with the ethical standards laid down in the 1964 Declaration of Helsinki and its later amendments. All participants provided informed consent prior to their inclusion in the study. Participants were selected from the Incidence of Cognitive Impairment in Cohorts with Longitudinal Evaluation in Parkinson's Disease (ICICLE-PD) study. The protocol has been described elsewhere [11], but in summary, we recruited 157 newly diagnosed patients with idiopathic PD, by United Kingdom PD Brain bank criteria [12], from community and outpatient clinics in Newcastle upon Tyne/Gateshead. Unrelated controls $(n=99)$, aged 45 years and over were recruited from the local community.

Exclusion criteria were: insufficient knowledge of English; dementia according to Movement Disorder Society (MDS) criteria [13] or significant memory impairment as evidenced by a Mini-Mental State Examination (MMSE) [14] score of $<24$ at presentation, drug-induced parkinsonism, progressive supranuclear palsy, multiple system atrophy or corticobasal degeneration.

Structured interviews included demographic data, neurologic examination, the Movement Disorders Society Unified PD Rating Scale part III (UPDRS-III) [15], Hoehn and Yahr stage [16] and neuropsychological tests covering all cognitive domains [17]. Performances on the individual tasks were transformed into $z$-scores and the average in each cognitive domain was calculated [18]. For data that were not normally distributed and could not be transformed appropriately, percentiles derived from a normal distribution were used to estimate cut-offs $1 \mathrm{SD}$ (16th percentile), 1.5
SD (7th percentile) and 2 SD (2nd percentile), therefore, the cut-offs give approximately the correct percentage of people impaired. For example, the pentagon score was assessed as 2 (shape includes 10 angles and clear intersection), 1 (two intersecting figures, one with five angles) or 0 (less acceptable copy); using corresponding percentiles from the control group, participants scoring 1 were classified as having impairment at the $1 \mathrm{SD}$ and $1.5 \mathrm{SD}$ level, and participants scoring 0 were classified as having impairment at the $2 \mathrm{SD}$ level [17]. Assessments were completed in an 'on' motor state. Clinical phenotypes of tremor dominant (TD) and postural instability/gait disorder (PIGD) were derived from the UPDRS scores [19]. PD medications were recorded and levodopa equivalent daily dose was calculated [20].

Using the MDS Task Force criteria [21], PD-MCI was diagnosed when patients scored 2 SD below normative means on at least two cognitive tests either in two different cognitive domains (attention, memory, language, executive and visuospatial functions) or two tests in the same domain. The choice of 2 SD cut-off was based on previous studies showing it gives greater diagnostic certainty [22] and optimal sensitivity and specificity [23]. As we had only one visuospatial test (pentagon copying from the MMSE), to be consistent with our previous studies [11,24], modified level II criteria were used. From our cohort of 157 patients with PD, 39 were diagnosed as PD-MCI. Seventy-four participants (42 controls, 23 PD-NC and 9 PD-MCI) did not have baseline DTI scans and in two patients (1 PD-NC and 1 PD-MCI) the baseline DTI scan had technical problems during acquisition. Nine controls had to be excluded as they met the criteria for MCI and three patients (1 PD-NC and 2 PD-MCI) were excluded retrospectively as at follow-up they were re-diagnosed as non-idiopathic PD. Therefore, the final baseline cohort consisted of 168 participants (48 controls, 93 PD-NC and 27 PD-MCI). At follow-up, two participants had died (1 PD-NC and 1 PD-MCI), twenty-nine did not complete the assessments (11 controls, 13 PD-NC and 5 PD-MCI), and the scanner had technical problems during the follow-up of two consecutive controls.

\section{MRI acquisition}

Participants had baseline and follow-up MRIs on the same 3T Intera Achieva scanner (Philips Medical Systems, Eindhoven, The Netherlands) with an eight-channel receiver head coil. A whole-brain standard sagittal T1-weighted volumetric scan was acquired using a magnetization prepared rapid gradient echo sequence: echo time $=4.6 \mathrm{~ms}$; repetition time $=9.6 \mathrm{~ms}$; inversion time $=4.6 \mathrm{~ms}$, flip angle $8^{\circ}$, sensitivity encoding factor $=2$; in-plane field of view $=240 \times 240 \mathrm{~mm}$; slice thickness $=1.2 \mathrm{~mm}$; voxel size $=1.15 \times 1.15 \mathrm{~mm}$. DTI acquisitions were based on a two-dimensional diffusion-weighted, spin-echo, echo 
planar imaging sequence with 59 slices: Repetition time $=6100 \mathrm{~ms}$; echo time $=70 \mathrm{~ms}$; flip angle $=90^{\circ}$; voxel size $=2.1 \times 2.1 \mathrm{~mm}$; slice thickness $=2.1 \mathrm{~mm}$; field of view $=270 \times 270 \mathrm{~mm}$. Diffusion weighting was performed in 64 directions (diffusion $b=1000 \mathrm{~s} / \mathrm{mm}^{2}$ ) and in six acquisitions without diffusion weighting $\left(b=0 \mathrm{~s} / \mathrm{mm}^{2}\right)$.

\section{MRI pre-processing}

Voxel-based analysis of the DTI data was performed using tract-based spatial statistics (TBSS) [25] in FSL (http:// www.fmrib.ox.ac.uk/fsl) 5.0.2 [26]. The baseline and follow-up total motion index were compared between groups: no group differences were demonstrated either at baseline or follow-up (Supplemental Data 1). After brain extraction, eddy current-induced distortion and subject movements were corrected using the Eddy FSL toolbox. FA and MD images were created by fitting a tensor model to the raw diffusion data. All FA images were aligned in the standard Montreal Neurological Institute 152 (MNI152) space using the nonlinear registration tool. The mean of all aligned FA images was created and thinned to build a mean FA skeleton representing the centres of all tracts common to all image volumes. The baseline skeleton was created using all subjects' baseline images, whereas the longitudinal skeleton included the baseline images of only those participants who completed the follow-up and their respective follow-up images all-together. Each aligned FA image volume was projected onto the common skeleton by filling the skeleton with FA values from the nearest relevant tract center. The resulting data were fed into the voxelwise cross-subject statistics. To estimate the WM microstructural change over 18 months for each participant, we subtracted each projected skeletonized FA image at follow-up from their respective baseline image. The difference images were then used in our statistical models. The same steps were followed for the MD images.

\section{Data analyses}

Image Analyses: Voxel-wise statistics were applied to the skeletonized FA and MD data using the randomize tool from FSL [27], which performed a 5000 permutation-based testing using threshold-free cluster enhancement $(p<0.05)$, corrected for multiple comparisons using the family-wise error correction. Group differences regarding FA and MD at baseline and interval changes at follow-up were tested using General Linear Model. The designs matrix consisted of t-contrasts with age, sex, education, levodopa equivalent daily dose and, when appropriate, scan intervals and disease duration as covariates for comparison between disease groups. White matter lesion volume (normalised to whole brain volume) was assessed using the method of Firbank et al. [28].
Clinical data: ANOVA (F) or two-sample Student's $t(t)$ test were used to compare means between groups. When ANOVA disclosed a significant main effect of group, post hoc differences were assessed using post hoc Bonferroni multiple-comparison tests. A Chi-square test $\left(x^{2}\right)$ was used for categorical data comparison. The relationships between baseline FA or MD with motor impairment and cognition were tested using multiple linear regression models, where baseline cognitive or motor scores were the dependent variables and FA or MD, the independent variables. These analyses were controlled for age, sex, education, disease duration and levodopa equivalent daily dose. However, when testing if baseline FA or MD were independent predictors of cognitive decline or worsening of motor impairment after 18 months, the follow-up cognitive or motor scores were used as the dependent variables, and the analyses were also controlled for baseline cognitive or motor scores and interval between visits. For all multiple regression analyses, we extracted the total brain baseline values of MD and FA using the 'fslmeants' tool from the FSL which extracts the average time series values of all selected voxels. All analyses were performed in STATA version 14.1, all tests were twotailed, significant results are reported at $p<0.05$. Due to the high inter-dependency of the FA and MD measures and the exploratory nature of this study, we did not apply Bonferroni corrections for multiple testing when investigating within group data.

\section{Results}

The baseline analyses were performed on the 168 participants (48 controls, 93 PD-NC and 27 PD-MCI) who completed the clinical assessment and had baseline DTI scans that passed quality control. After a mean of 18.8 (SD 1.6) months, 135 (35 controls, 79 PD-NC and 21 PD-MCI) participants completed the follow-up clinical assessment and were re-scanned successfully. The baseline demographics and clinical characteristics of participants are summarized in Table 1.

The sex distribution between groups was not significantly different. Patients with PD-MCI were significantly older than patients with PD-NC. Education was significantly different among groups, however, when interrogation with post hoc Bonferroni pairwise tests, no significant differences were found. The proportion of WML was not significantly different between groups. Disease duration and motor impairment was similar in both groups. In addition, levodopa equivalent daily dose intake was not significantly different among groups. Compared to PD-NC, patients with PD-MCI were significantly more affected as evidenced by higher Hoehn and Yahr stages. The clinical 
Table 1 Baseline demographics and clinical characteristics

\begin{tabular}{llllll}
\hline Characteristic & Control $(n=48)$ & PD-NC $(n=93)$ & PD-MCI $(n=27)$ & Statistic & $p$ \\
\hline Female, $\%$ & $40 \%$ & $34 \%$ & $30 \%$ & $x^{2}(2)=0.8$ & 0.671 \\
Age, years & $66.0(7.9)$ & $64.3(10.8)$ & $70.5(8.1)$ & $f(2,165)=4.3$ & $0.015^{\mathrm{a}}, 0.011^{\mathrm{d}}$ \\
Education, years & $13.7(3.7)$ & $13.5(3.8)$ & $11.6(4.1)$ & $f(2,165)=3.1$ & $0.046^{\mathrm{a}, \mathrm{e}}$ \\
Proportion of WML & $0.003(0.01)$ & $0.004(0.01)$ & $0.007(0.01)$ & $f(2,165)=1.8$ & 0.174 \\
Time to follow-up, months & $20.0(1.7)$ & $18.2(1.3)$ & $19.0(1.5)$ & $f(2,165)=20.1$ & $<0.001^{\mathrm{a}},<0.001^{\mathrm{b}}, 0.033^{\mathrm{c}}$ \\
Duration of PD, months & - & $6.4(0.5)$ & $5.6(0.7)$ & $t(118)=0.7$ & 0.466 \\
UPDRS-III & - & $25.9(1.1)$ & $29.2(2.2)$ & $t(118)=1.4$ & 0.176 \\
Hoehn and Yahr & - & $1.9(0.1)$ & $2.3(0.1)$ & $\mathrm{t}(118)=2.4$ & 0.018 \\
Levodopa equivalent dose, mg & - & $161.0(13.7)$ & $214.0(24.9)$ & $t(118)=1.8$ & 0.069 \\
Clinical phenotype, $\%$ & - & $44 \%$ & & $x^{2}(2)=0.4$ & 0.813 \\
Postural instability/gait difficulty & - & $40 \%$ & $46 \%$ & & \\
Tremor dominance & - & $15 \%$ & $35 \%$ & $18 \%$ & \\
Indeterminate & - & & & & \\
\hline
\end{tabular}

Values expressed as mean (SD)

${ }^{\mathrm{a}}$ ANOVA, Post hoc Bonferroni pairwise tests: ${ }^{\mathrm{b}}$ Controls versus PD-NC, ${ }^{\mathrm{c} C o n t r o l s ~ v e r s u s ~ P D-M C I, ~}{ }^{\mathrm{d}} \mathrm{PD}-\mathrm{NC}$ versus PD-MCI, ${ }^{\mathrm{e}}$ no significant results on pairwise tests

phenotype distribution was not significantly different between the two groups of patients with PD (Table 1).

\section{Baseline diffusion tensor imaging parameters}

Patients with PD-MCI and PD-NC had significantly higher white matter MD compared with controls in widespread regions, involving bilaterally the corona radiata, internal and external capsule, corpus callosum, inferior and superior fronto-occipital fasciculi, forceps minor, cingula, superior and inferior longitudinal fasciculi (Fig. 1). There were no significant differences in MD between PD-MCI and PD-NC at baseline. In terms of FA, patients with PDMCI had significant lower FA than controls in posterior aspect of the inferior fronto-occipital fasciculi, superior and inferior longitudinal fasciculi, forceps major, corticospinal tracts and cingula (Fig. 1). No significant FA differences between the remaining groups.

\section{Relationship between baseline diffusion tensor imaging parameters and cognition}

At baseline, among the patients with PD-MCI, higher MD was significantly related to lower attention and executive function scores. The relationships between the other cognitive functions or general cognition with FA or MD baseline values were not significant among any of the study groups (Table 2).

\section{Relationship between baseline diffusion tensor imaging parameters and motor function}

Within the combined group of PD (PD-NC and PD-MCI), the relationships between baseline motor impairment with FA or MD were not significant (Table 3).

Regarding the motor phenotypes, there were no significant differences in terms of baseline FA $(F(2,117) 0.03, p$ $0.972)$ or $\operatorname{MD}(F(2,117) 0.82, p 0.443)$ values.

\section{Longitudinal diffusion tensor imaging parameters}

At follow-up, there were significant changes in both DTI parameters when compared to baseline, with FA decreasing and $\mathrm{MD}$ increasing significantly. However, compared to PD-NC, the PD-MCI group showed significant MD changes, increasing mainly in their frontal regions (Fig. 2). All these changes followed a bilateral and symmetric pattern. There were no significant differences in the change in MD in the PD groups compared with controls. Similarly, FA changes were not significantly different between groups.

\section{Diffusion tensor imaging parameters as predictors of decline in cognition}

At follow-up, 15\% (12 out of 79) of PD-NC progressed to MCI and 14\% (3 out of 21) of those participants with baseline PD-MCI converted to dementia. Lower baseline values of FA were significantly related with greater decline in executive function scores among controls. Within the 

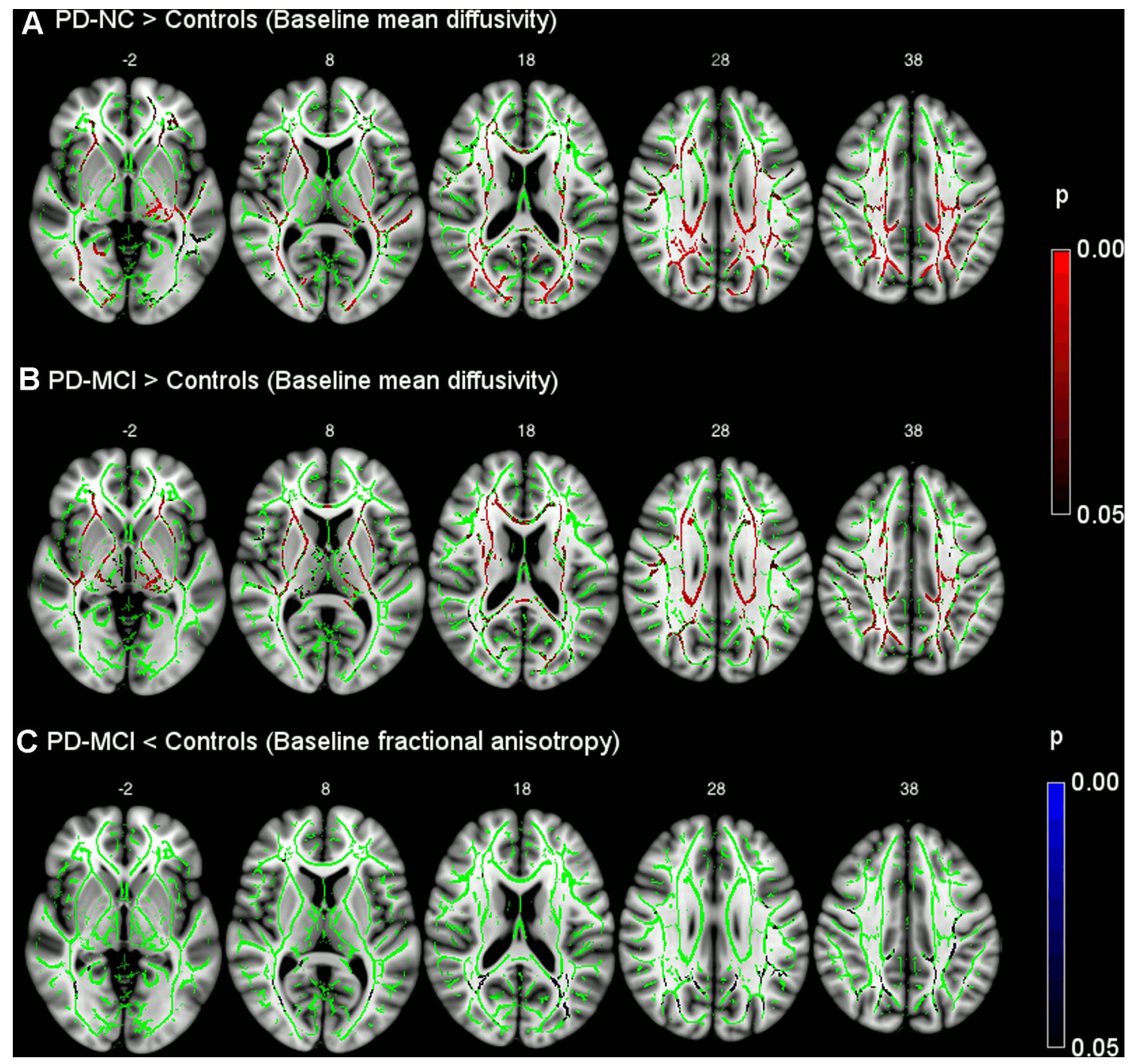

Fig. 1 Differences in baseline mean diffusivity. TBSS: regions at baseline of increased mean diffusivity and reduce fractional anisotropy $(p<0.05$ corrected) (a). Increased mean diffusivity between patients with PD-NC and controls (b) between patients with PDMCI and controls. c Reduced fractional anisotropy between patients

participants with either PD-NC or PD-MCI, MD and FA were not significantly related to cognitive scores (Table 4). Among those patients with PD-NC, FA [ $\beta$ (95\% CI) 0.001 $(-0.007 ; 0.009), p 0.787]$ and $\mathrm{MD}[\beta(95 \% \mathrm{CI})-0.004$ $(-0.017 ; 0.010), p 0.605]$ values were not predictors of progression to MCI.

\section{Diffusion tensor imaging parameters as predictors of decline in motor impairment}

Within the combined group of PD (PD-NC and PD-MCI), higher MD baseline values were significant predictors of motor impairment worsening, however, the relationship with PD-MCI and controls. TBSS results are shown overlaid on an MNI152 template and the mean FA skeleton (green). Significantly different voxels of increased MD are shown in red shades and those of reduced FA, in blue. $Z$-coordinates are displayed

between baseline FA and motor impairment was not significant (Table 3 ).

Regarding the motor phenotypes, there were no significant differences in terms of longitudinal changes in FA $[F(2$, 97) $1.09, p 0.341]$ or $\mathrm{MD}[F(2,97) 1.59, p 0.210]$ values.

\section{Discussion}

This study showed that MD is a significant predictor of worsening motor function in early Parkinson's disease over 18 months. We also demonstrated that MD differed between patients with Parkinson's disease and controls even soon after diagnosis. Furthermore, in patients with PD-MCI, 
Table 2 Multiple linear regression analyses examining the relationship between fractional anisotropy and mean diffusivity with cognitive in patients with early Parkinson's disease and controls

\begin{tabular}{|c|c|c|c|c|c|}
\hline \multirow[t]{2}{*}{ Tests } & \multirow{2}{*}{$\begin{array}{l}\text { Baseline test scores } \\
\text { Mean (SD) }\end{array}$} & \multicolumn{2}{|c|}{ Baseline fractional anisotropy } & \multicolumn{2}{|l|}{ Baseline mean diffusivity } \\
\hline & & $\beta(95 \% \mathrm{CI})$ & $\mathrm{p}$ & $\beta(95 \% \mathrm{CI})\left(\times 10^{3}\right)$ & $p$ \\
\hline \multicolumn{6}{|l|}{ Controls } \\
\hline MMSE & $29.35(0.81)$ & $8.50(-5.59 ; 22.60)$ & 0.230 & $-5.47(-15.52 ; 4.59)$ & 0.279 \\
\hline Attention & $0.00(0.80)$ & $0.57(-12.88 ; 14.03)$ & 0.932 & $0.10(-9.51 ; 9.71)$ & 0.983 \\
\hline Executive function & $-0.01(0.70)$ & $-7.97(-19.33 ; 3.39)$ & 0.164 & $7.54(-0.35 ; 15.42)$ & 0.061 \\
\hline Language & $0.00(0.80)$ & $3.09(-11.12 ; 17.30)$ & 0.663 & $1.83(-8.40 ; 12.06)$ & 0.720 \\
\hline Memory & $0.00(0.77)$ & $4.88(-8.52 ; 18.27)$ & 0.466 & $-5.88(-15.35 ; 3.59)$ & 0.217 \\
\hline Visuospatial function & $0.00(1.00)$ & $-3.13(-21.12 ; 14.86)$ & 0.727 & $0.42(-12.48 ; 13.31)$ & 0.948 \\
\hline \multicolumn{6}{|l|}{ PD-NC } \\
\hline MMSE & $28.91(1.05)$ & $-8.47(-23.37 ; 6.43)$ & 0.262 & $4.79(-4.81 ; 14.38)$ & 0.324 \\
\hline Attention & $-0.48(1.04)$ & $3.14(-11.27 ; 17.55)$ & 0.666 & $2.78(-6.48 ; 12.04)$ & 0.552 \\
\hline Executive function & $-0.43(0.70)$ & $1.21(-7.70 ; 10.12)$ & 0.787 & $0.68(-5.04 ; 6.41)$ & 0.813 \\
\hline Language & $-0.05(0.87)$ & $-0.49(-13.25 ; 12.27)$ & 0.940 & $5.10(-3.02 ; 13.22)$ & 0.215 \\
\hline Memory & $-0.47(0.93)$ & $7.84(-4.58 ; 20.25)$ & 0.213 & $-5.48(-13.35 ; 2.39)$ & 0.170 \\
\hline Visuospatial function & $0.12(0.73)$ & $2.65(-7.73 ; 13.03)$ & 0.613 & $-2.54(-9.20 ; 4.12)$ & 0.450 \\
\hline \multicolumn{6}{|l|}{ PD-MCI } \\
\hline MMSE & $27.81(1.69)$ & $3.66(-35.53 ; 42.86)$ & 0.847 & $-5.35(-30.53 ; 19.84)$ & 0.663 \\
\hline Attention & $-3.23(2.83)$ & $47.12(-17.21 ; 111.45)$ & 0.142 & $-42.63(-81.72 ;-3.54)$ & 0.034 \\
\hline Executive function & $-1.80(1.21)$ & $20.68(-6.29 ; 47.65)$ & 0.125 & $-20.43(-36.26 ;-4.60)$ & 0.014 \\
\hline Language & $-0.68(1.10)$ & $-4.26(-33.13 ; 24.61)$ & 0.759 & $11.55(-6.57 ; 29.66)$ & 0.196 \\
\hline Memory & $-2.72(1.70)$ & $8.12(-29.76 ; 45.99)$ & 0.660 & $-10.20(-34.29 ; 13.89)$ & 0.387 \\
\hline Visuospatial function & $-1.11(2.54)$ & $-39.48(-100.04 ; 21.07)$ & 0.189 & $12.41(-28.01 ; 52.83)$ & 0.529 \\
\hline
\end{tabular}

Each analysis was controlled for age, sex, education, disease duration and levodopa equivalent daily dose

Table 3 Multiple linear regression analyses examining the relationship between fractional anisotropy and mean diffusivity with motor impairment in patients with early Parkinson's disease

\begin{tabular}{lll}
\hline UPDRS-III & $\beta(95 \%$ CI $)$ & $p$ \\
\hline Baseline $^{\mathrm{a}}$ & & \\
Fractional anisotropy & $-3.92(-129.14 ; 121.29)$ & 0.951 \\
$\quad$ Mean diffusivity $\left(\times 10^{3}\right)$ & $16.90(-63.57 ; 97.38)$ & 0.678 \\
Follow-up $^{\mathrm{b}}$ & & \\
Fractional anisotropy $_{\text {Mean diffusivity }\left(\times 10^{3}\right)}$ & $-102.51(-215.90 ; 10.88)$ & 0.076 \\
\hline
\end{tabular}

${ }^{a}$ Analyses were controlled for age, sex, education, disease duration and levodopa equivalent daily dose

${ }^{\mathrm{b}}$ Analyses were controlled for age, sex, education, disease duration, levodopa equivalent daily dose, baseline motor scores and interval between visits

increased mean diffusivity was significantly associated with attention and executive function impairments, and frontal MD increased significantly faster when compared to those with PD-NC.

The current results confirm our principal hypothesis, and reinforce previous cross-sectional observations [18] of greater cortical thinning in the frontal and temporal cortices in PD-MCI compared to cognitively normal patients. It suggests that both cortical integrity and connectivity of the frontal lobe are vital for successful cognitive function [29]. The focal pattern of frontal white matter deterioration in PD-MCI raises the possibility that there is a synergistically detrimental effect of disconnection and concurrent cortical atrophy [18]. However, such causality cannot yet be confirmed with our current results. Interestingly, both white matter microstructure and cortical thickness differences between the two PD sub-groups only became evident after 18 months.

We demonstrated that in early PD-MCI, higher values of MD are associated with lower attention and executive function scores. For these analyses we did not correct for multiple testing, however, our weak but significant $p$ values are concordant with the previously published research demonstrating that even slight changes in the white matter might be a contributing factor to the Parkinson's disease dysexecutive syndrome [7]. Therefore, by accepting the null hypothesis, we would most likely be incurring in error type II as it is well known that the Bonferroni's correction is too conservative. Our findings support the hypothesis that disruption of prefrontal connections to the basal ganglia, represented by white matter microstructural changes are implicated in some of the cognitive 


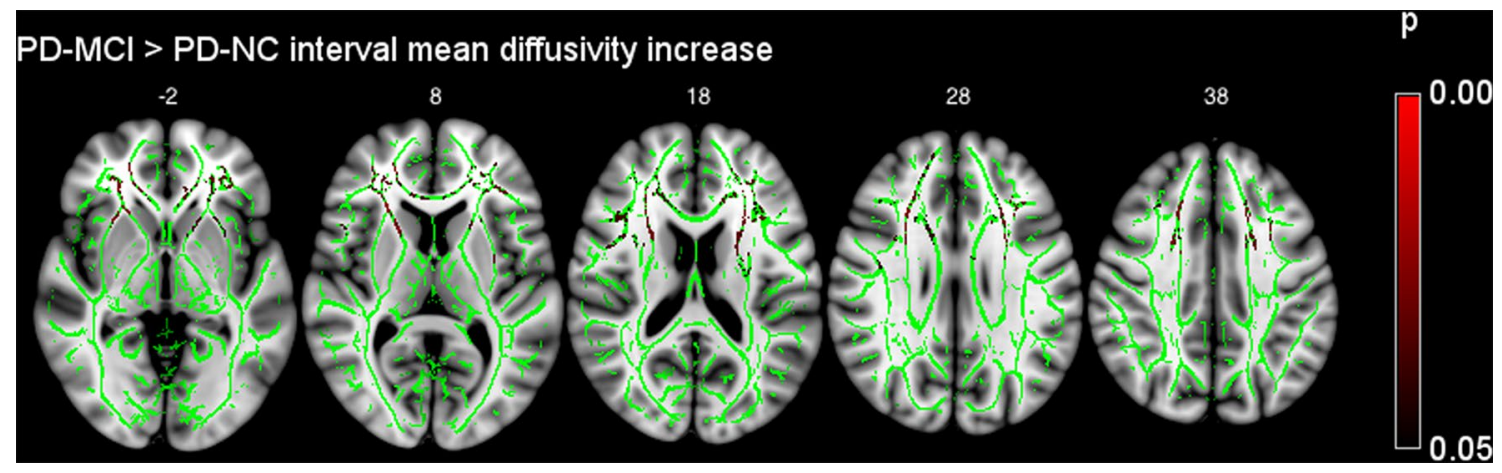

Fig. 2 Differences in longitudinal mean diffusivity. TBSS: regions at follow-up of increased mean diffusivity interval changes $(p<0.05$ corrected) between patients with PD-MCI compared to those with
PD-NC. TBSS results are shown overlaid on an MNI152 template and the mean FA skeleton (green). Significantly different voxels of increased MD are shown in red shades. Z-coordinates are displayed

Table 4 Multiple regression analyses of fractional anisotropy or mean diffusivity as independent predictors of cognitive scores in patients with early Parkinson's disease and controls

\begin{tabular}{|c|c|c|c|c|c|}
\hline \multirow[t]{2}{*}{ Tests } & \multirow{2}{*}{$\begin{array}{l}\text { Follow-up test scores } \\
\text { Mean (SD) }\end{array}$} & \multicolumn{2}{|c|}{ Baseline fractional anisotropy } & \multicolumn{2}{|l|}{ Baseline mean diffusivity } \\
\hline & & $\beta(95 \% \mathrm{CI})$ & $p$ & $\overline{\beta(95 \% \mathrm{CI})\left(\times 10^{3}\right)}$ & $p$ \\
\hline \multicolumn{6}{|l|}{ Controls } \\
\hline MMSE & $29.83(0.45)$ & $2.26(-7.80 ; 12.32)$ & 0.651 & $-0.23(-6.78 ; 6.33)$ & 0.944 \\
\hline Attention & $-0.18(0.86)$ & $2.12(-10.84 ; 15.07)$ & 0.742 & $2.48(-5.94 ; 10.90)$ & 0.553 \\
\hline Executive function & $0.08(0.63)$ & $10.23(1.38 ; 19.07)$ & 0.025 & $-3.76(-10.02 ; 2.50)$ & 0.231 \\
\hline Language & $0.80(0.63)$ & $-1.92(-22.36 ; 18.51)$ & 0.849 & $0.57(-12.73 ; 13.87)$ & 0.931 \\
\hline Memory & $-0.33(0.85)$ & $4.03(-10.09 ; 18.15)$ & 0.565 & $1.08(-8.33 ; 10.49)$ & 0.818 \\
\hline Visuospatial function & $0.26(0.00)$ & $1.59(-9.63 ; 12.81)$ & 0.776 & $-0.41(-7.73 ; 6.91)$ & 0.911 \\
\hline \multicolumn{6}{|l|}{ PD-NC } \\
\hline MMSE & $28.95(1.30)$ & $-2.51(-20.89 ; 15.87)$ & 0.786 & $5.92(-5.57 ; 17.40)$ & 0.308 \\
\hline Attention & $-0.85(1.37)$ & $4.05(-9.70 ; 17.81)$ & 0.559 & $-0.57(-9.32 ; 8.19)$ & 0.898 \\
\hline Executive function & $-0.23(0.88)$ & $1.56(-9.96 ; 13.07)$ & 0.789 & $-2.09(-9.39 ; 5.21)$ & 0.571 \\
\hline Language & $0.03(0.69)$ & $3.35(-6.78 ; 13.49)$ & 0.511 & $-1.96(-8.44 ; 4.52)$ & 0.548 \\
\hline Memory & $-0.64(1.19)$ & $-8.16(-21.55 ; 5.23)$ & 0.228 & $6.73(-1.56 ; 15.02)$ & 0.110 \\
\hline Visuospatial function & $-0.31(1.57)$ & $7.92(-14.57 ; 30.41)$ & 0.485 & $1.78(-12.46 ; 16.02)$ & 0.804 \\
\hline \multicolumn{6}{|l|}{ PD-MCI } \\
\hline MMSE & $27.00(1.79)$ & $27.43(-15.88 ; 70.74)$ & 0.197 & $-21.45(-51.05 ; 8.16)$ & 0.143 \\
\hline Attention & $-3.64(3.40)$ & $4.64(-49.77 ; 59.05)$ & 0.858 & $-4.18(-42.65 ; 34.29)$ & 0.820 \\
\hline Executive function & $-1.77(0.99)$ & $9.79(-10.54 ; 30.11)$ & 0.321 & $-10.29(-24.03 ; 3.44)$ & 0.131 \\
\hline Language & $-0.60(1.08)$ & $4.49(-19.30 ; 28.27)$ & 0.690 & $-3.57(-20.49 ; 13.35)$ & 0.656 \\
\hline Memory & $-2.91(1.60)$ & $14.53(-21.53 ; 50.59)$ & 0.404 & $-4.73(-30.95 ; 21.49)$ & 0.706 \\
\hline Visuospatial function & $-3.25(3.72)$ & $62.76(-27.61 ; 153.13)$ & 0.159 & $-29.54(-92.55 ; 33.47)$ & 0.333 \\
\hline
\end{tabular}

Each analysis was controlled for baseline test scores, age, sex, education, levodopa equivalent daily dose, disease duration and interview interval

features of PD. According to the classical "parallel loop" model, homologous circuits connect frontal cortex to basal ganglia and thalamus [30]. These circuits are related to motor and oculomotor function, cognition and emotional regulation. Particularly, the dorsolateral prefrontal circuit that has been linked with PD, affecting executive function $[31,32]$. We failed to demonstrate that MD and FA values are predictors of cognitive decline. It may be that
18 months of interval was not long enough to demonstrate such relationship.

A meta-analyses [33] on diffusion tensor imaging in PD demonstrated that FA and MD values could distinguish patients with PD from healthy controls in five regions: the substantia nigra, the corpus callosum, the cingulate and temporal cortices, and the corticospinal tracts, the latter, being associated with increased FA and decreased MD, suggesting 
possible brain reorganization. Furthermore, MD values only could differentiate PD from controls in the putamen, globus pallidum and internal capsules. We demonstrated that even at early stages of $\mathrm{PD}$, mean diffusivity is significantly higher in the corona radiata, internal and external capsules, corpus callosum, inferior and superior fronto-occipital fasciculi, forceps minor, cingula, superior and inferior longitudinal fasciculi, and FA is reduced in the posterior aspect of the inferior fronto-occipital fasciculi, superior and inferior longitudinal fasciculi, forceps major, corticospinal tracts and cingula.

Ofori et al. [34] investigated diffusional longitudinal changes within the substantia nigra in Parkinson's disease using an advanced bi-tensor diffusion model and demonstrated that baseline elevated free-water levels in the posterior substantia nigra predicted 1 year worsening of bradykinesia and general cognitive decline. They suggested that the progressive increase of free-water levels might be related to the characteristic degeneration of dopaminergic cells in the substantia nigra. Our study demonstrated that baseline white matter MD values predicted 18 months worsening of motor symptoms. Although we did not specifically measure substantia nigra free water, it is possible that the findings observed by Ofori et al. [34] and ours are interconnected phenomena representing deficits of the substantia nigra functional connectivity. Few other studies showed significant correlations between motor impairment in PD and substantia nigra diffusional changes [34-36]. Rae et al. [7] identified correlation between impairment in motor function and reduced fractional anisotropy beyond the substantia nigra, in the white matter. Their findings were based on a crosssectional sample and no correlations were found between mean diffusivity and motor features. In our study we failed to identify cross-sectional associations between white matter tract microstructure and motor disability, however, we demonstrated that baseline increased MD was predictive of worsening of motor symptoms. This suggests that direct presynaptic deposition of PD-related neurodegenerative pathologies could lead to axonal degeneration impairing structural connectivities. A combined DTI and graph analysis study found alterations in the structural connectivity of several motor and non-motor regions present already at an early stage of disease [37].

Given the well-established differences in disease progression according to the different motor phenotypes of PIGD and TD in Parkinson's disease [38], we investigated the relationship between these phenotypes and DTI metrics. No significant differences were found at baseline or 18 months later: the longitudinal changes in MD and FA were not significantly different according to phenotype. Similar TBSS baseline findings were also previously demonstrated [39], however, when probabilistic tractography was used, increased MD was demonstrated in tracts connecting the right premotor cortex and right inferior parietal lobule in TD compared to PIGD. Most previous studies that have reported FA and MD differences according to clinical phenotypes used tractography or region of interest rather than TBSS [40], which is beyond the scope of our study.

The TBSS voxelwise analysis that we applied is an automated observer-independent technique which reduces potential bias because it does not require a priori selection of regions of interest. Furthermore, no arbitrary spatial smoothing is necessary, minimizing misalignment and misregistration, increasing the sensitivity and interpretability of findings [25]. However, as a drawback TBSS has the disadvantage of only providing group comparisons of the DTI parameters at the major white matter bundles without much specificity, therefore, not informing which regions are connected by individual fibre tracts; furthermore, voxels further from tract centres decrease weighting in the average of voxels projected to that location, thereby reducing the sensitivity to detect changes in such locations [41]. This potential lack of TBSS sensitivity added to the short disease duration of our patients with PD $(6.0 \pm 4.6$ months $)$ might have impacted in our failure to demonstrate baseline differences between the PD-NC and PD-MCI in terms of white matter integrity, even though the two patients groups were significantly different in terms of disease severity and cognitive status. The cross-sectional study which used the most comparable methodology to ours [8] involving TBSS and grouping patients according to formal criteria to diagnose PD-MCI, also found that all PD groups had higher MD values compared to controls. However, in contrast, their patients with PD and cognitive impairment had higher MD values than those with PD-NC. This might be explained by their patients' longer disease duration than in our study (5.8 \pm 5.1 years). In line with our findings, a longitudinal study [42] demonstrated that PD patients and matched controls exhibited DTI changes over time. They suggested that DTI PD-specific changes over 1 year did not extend beyond the normal age-associated change. Unfortunately, among their 19 participants with PD, only four had MCI, precluding subgroup analyses. Another longitudinal study using TBSS [43], which followed patients with PD for 2 years, did not demonstrate any significant time or group effects in FA between PD and controls, or between PD-NC and PD-MCI. The small number of participants $(n 25)$ will have reduced their power to detect subgroup differences.

In our study, white matter lesions were not significantly different between groups. We did not include white matter hyperintensity volume as a covariate in our analyses as we do not consider white matter hyperintensities as a confounder but part of a spectrum of the white matter changes. Evidence from a large representative population-based autopsy driven study [44] has shown that MRI detection of white matter hyperintensities is less sensitive than pathology 
and false negative MRIs are associated with milder pathology. Furthermore, the same group [45] showed that the prevalence of white matter lesions is as high as $94 \%$ regardless of dementia status. Abnormal DTI measures in white matter tracts connecting key regions affected by Parkinson's disease pathology can also be affected by small-vessel disease and white matter lesions, thereby making it difficult to disentangle vascular from non-vascular pathological processes in Parkinson's disease and PD-MCI. Our main interest was to characterise the trajectories of DTI metrics in Parkinson's disease irrespective of the pathological process that is contributing to the white matter changes, hence not including white matter lesions as a covariate.

Our diffusion imaging protocol prioritized a large number of gradient directions (64), enabling greater angular resolution, therefore, a more accurate FA estimation [7]. However, we demonstrated only modest FA differences at baseline and did not demonstrate FA changes at follow-up. It may be that fractional anisotropy reduction only occurs later in the course of disease progression. Our cohort comprised newly diagnosed Parkinson's disease cases who had very short disease duration. It is possible that MD measurements are inherently more sensitive than FA for detecting differences in white matter microstructure as MD is more uniform across the brain than FA, and therefore, less affected by registration errors in relation to voxel size [43]. Furthermore, while MD characterizes the mean diffusion along both axonal and radial orientations, revealing how restricted diffusion is within the white matter, FA characterizes diffusion in the axonal orientation relative to radial orientations, representing the degree of directional diffusion $[7,46]$. If $\mathrm{MD}$ is sensitive but directionally non-specific, any process leading to white matter microstructural degeneration would cause MD to invariably increase, whereas FA provides more specific information as it depends on the underlying arrangement of the fibres [46]. Even so, some authors also consider FA to be non-specific because decreases may result from either increased radial (perpendicular) diffusivity or reduced axial (parallel) diffusivity [47].

This study has some limitations. First, our patients with PD-MCI were significantly older with the more advanced disease than the PD-NC. Even though we adjusted the analyses for age, this is a potential bias as older patients with PD have a faster rate of disease progression than those at younger ages [48]. We, therefore, performed a series of sensitivity analyses selecting subsets of older and with more advanced disease patients with PD-NC and found that our results remained robust (Online Table e-1). Second, some participants left the study before the second MRI acquisition. As the TBSS implementation of its general linear model does not accommodate incomplete datasets, we could not adjust for missing data by means or imputation. However, it is unlikely to have significantly influenced our main results, as there were no significant differences between those who left the study and those who continued regarding demographics and clinical features (Online Table e-2). Third, the gap between MRI studies was significantly higher among controls, and they had the highest drop-out rate. This probably influenced our power to detect significant differences. Finally, it should also be noted that PD patients were assessed while taking their medication which might have influenced some aspects of cognition [49], but is unlikely to have significantly altered the diffusion metrics [7]. However, in contrast to most other studies, we included levodopa equivalent dose in our analyses [20].

This study with a prospective longitudinal design, allowed us to evaluate white matter microstructure, at baseline and at 18 months, to assess the potential for DTI in predicting cognitive decline and worsening of motor function. Our results are derived from a well-characterized, large cohort of patients with early PD, with and without MCI based on consensus MDS criteria. Our images were gathered all at one site and analyzed using a TBSS approach which enables a rather unbiased analysis. We demonstrated that WM microstructural changes can differentiate between Parkinson's disease and controls even in early stages of disease and MD is associated with attention and executive function impairment in PD-MCI. Furthermore, MD is a predictor of future worsening of motor impairment. We also showed that in PD-MCI, the frontal mean diffusivity increases significantly more in PD-MCI than in PD without MCI. Our findings support the hypothesis that MD represents an important correlate of cognitive function and is a predictor of motor decline in PD. As such diffusion tensor imaging is potentially a useful tool in stratification of patients into clinical trials to monitor the impact of treatment on motor and cognitive features.

Author contributions TM: Imaging processing, concluded statistical analyses, manuscript draft and revision. LS: Data analyses, manuscript revision. EM: Manuscript revision. GW: Manuscript revision. MF: Manuscript revision. RAL: Study coordination, participant recruitment, data collection, manuscript revision. AJY: Study coordination, participant recruitment, clinical assessment, data collection and manuscript revision. GWD: Study coordination, participant recruitment, clinical assessment, data collection and manuscript revision. AMO: Manuscript revision. TKK: Study coordination, participant recruitment, clinical assessment, data collection and manuscript revision. DJB: Principal investigator and co-applicant for the funding grant. He was involved in the study design and manuscript revision. JBR: Data acquisition and manuscript revision. RAB: Principal investigator and co-applicant for the funding grant. He was involved in the study design and manuscript revision. DB: Chief investigator and main applicant for the funding grant. He was involved with the study design, supervision and manuscript revision. JTO: Principal investigator and co-applicant for the funding grant. He was involved in the study design, supervision and manuscript revision.

Funding ICICLE-PD was funded by Parkinson's UK (J-0802, G-1301, G-1507) and supported by the Lockhart Parkinson's Disease Research 
Fund, NIHR (National Institute for Health Research) (RG64473); NIHR Biomedical Research Unit in Dementia at Cambridge University Hospitals NHS (National Health Service) Foundation Trust and the University of Cambridge; and NIHR Biomedical Research Unit in Dementia at Newcastle upon Tyne Hospitals NHS Foundation Trust and Newcastle University. TM is is funded by an Academic Clinical Fellow from NIHR. LS is supported by Alzheimer's Research UK (ARUK-SRF2017B-1). EM is in receipt of the Gates Cambridge scholarship. JBR is supported by the Wellcome Trust (103838) and Medical Research Council (MC-A060-5PQ30).

\section{Compliance with ethical standards}

Conflicts of interest Dr Thais Minett reports no disclosure. Dr Li Su reports no disclosure. Elijah Mak reports no disclosure. Dr Guy Williams reports no disclosure. Dr Michael Firbank reports no disclosure. Dr Rachael (A) Lawson reports no disclosure. Dr Alison J. Yarnall has received honoraria from Teva-Lundbeck and sponsorship from Teva-Lundbeck, UCB, GlaxoSmithKline, Genus and Abbvie for attending conferences. Dr Gordon W. Duncan reports no disclosure. Dr Adrian M. Owen reports no disclosure. Dr Tien K. Khoo reports no disclosure. Prof David J. Brooks reports no disclosure. Prof James (B) Rowe received grants from NIHR, Evelyn Trust, McDonnell Foundation, ARUK, PSP Association, AZ-Medimmune and Janssen, but no personal financial remuneration or consultancies or other conflict-ofinterest arising from these. Prof David Burn reports no disclosure. Prof John T. O'Brien reports no disclosure. Prof Roger A Barker received grants from Parkinson's UK, NIHR, Cure Parkinson's Trust, Evelyn Trust, Rosetrees Trust, MRC and EU along with payment for advisory board attendance from Oxford Biomedica and LCT, and honoraria from Wiley and Springer. Prof David Burn reports no disclosure.

Ethical approval The study was approved by the Newcastle and North Tyneside Research Ethics Committee and has, therefore, been performed in accordance with the ethical standards laid down in the 1964 Declaration of Helsinki and its later amendments. All participants provided informed consent prior to their inclusion in the study.

Disclaimer This article presents independent research funded by Parkinson's UK and the National Institute for Health Research. The views expressed are those of the authors and not necessarily those of the NHS, Parkinson's UK the National Institute for Health Research or the Department of Health.

\section{References}

1. Hobson P, Meara J (2004) Risk and incidence of dementia in a cohort of older subjects with Parkinson's disease in the United Kingdom. Mov Disord 19:1043-1049

2. Hely MA, Reid WG, Adena MA, Halliday GM, Morris JG (2008) The Sydney multicenter study of Parkinson's disease: the inevitability of dementia at 20 years. Mov Disord 23(6):837-844. https ://doi.org/10.1002/mds.21956

3. Foltynie T, Brayne CE, Robbins TW, Barker RA (2004) The cognitive ability of an incident cohort of Parkinson's patients in the UK. The CamPaIGN study. Brain 127(Pt 3):550-560. https://doi. org/10.1093/brain/awh067

4. Janvin CC, Larsen JP, Aarsland D, Hugdahl K (2006) Subtypes of mild cognitive impairment in Parkinson's disease: progression to dementia. Mov Disord 21(9):1343-1349. https://doi.org/10.1002/ mds. 20974
5. Bohnen NI, Albin RL (2011) White matter lesions in Parkinson disease. Nat Rev Neurol 7(4):229-236. https://doi.org/10.1038/ nrneurol.2011.21

6. Lee SJ, Kim JS, Yoo JY, Song IU, Kim BS, Jung SL, Yang DW, Kim YI, Jeong DS, Lee KS (2010) Influence of white matter hyperintensities on the cognition of patients with Parkinson disease. Alzheimer Dis Assoc Disord 24(3):227-233. https://doi. org/10.1097/WAD.0b013e3181d71a13

7. Rae CL, Correia MM, Altena E, Hughes LE, Barker RA, Rowe JB (2012) White matter pathology in Parkinson's disease: the effect of imaging protocol differences and relevance to executive function. Neuroimage 62(3):1675-1684. https://doi.org/10.1016/j. neuroimage.2012.06.012

8. Melzer TR, Watts R, MacAskill MR, Pitcher TL, Livingston L, Keenan RJ, Dalrymple-Alford JC, Anderson TJ (2013) White matter microstructure deteriorates across cognitive stages in Parkinson disease. Neurology 80(20):1841-1849. https://doi. org/10.1212/WNL.0b013e3182929f62

9. Halliday GM, Leverenz JB, Schneider JS, Adler CH (2014) The neurobiological basis of cognitive impairment in Parkinson's disease. Mov Disord 29(5):634-650. https://doi.org/10.1002/ mds. 25857

10. Duncan GW, Firbank MJ, Yarnall AJ, Khoo TK, Brooks DJ, Barker RA, Burn DJ, O'Brien JT (2016) Gray and white matter imaging: a biomarker for cognitive impairment in early Parkinson's disease? Mov Disord 31(1):103-110. https://doi. org/10.1002/mds.26312

11. Yarnall AJ, Breen DP, Duncan GW, Khoo TK, Coleman SY, Firbank MJ, Nombela C, Winder-Rhodes S, Evans JR, Rowe JB, Mollenhauer B, Kruse N, Hudson G, Chinnery PF, O'Brien JT, Robbins TW, Wesnes K, Brooks DJ, Barker RA, Burn DJ, Group I-PS (2014) Characterizing mild cognitive impairment in incident Parkinson disease: the ICICLE-PD study. Neurology 82(4):308316. https://doi.org/10.1212/WNL.0000000000000066

12. Gibb WRG, Lees AJ (1988) The relevance of the Lewy body to the pathogenesis of idiopathic Parkinson's disease. J Neurol Neurosurg Psychiatry 51:745-752

13. Emre M, Aarsland D, Brown R, Burn DJ, Duyckaerts C, Mizuno Y, Broe GA, Cummings J, Dickson DW, Gauthier S, Goldman J, Goetz C, Korczyn A, Lees A, Levy R, Litvan I, McKeith I, Olanow W, Poewe W, Quinn N, Sampaio C, Tolosa E, Dubois B (2007) Clinical diagnostic criteria for dementia associated with Parkinson's disease. Mov Disord 22(12):1689-1707. https://doi. org/10.1002/mds.21507 (quiz 1837).

14. Folstein MF, Folstein SE, McHugh PR (1975) "Mini-mental state". A practical method for grading the cognitive state of patients for the clinician. J Psychiatr Res 12(3):189-198

15. Goetz CG, Tilley BC, Shaftman SR, Stebbins GT, Fahn S, Martinez-Martin P, Poewe W, Sampaio C, Stern MB, Dodel R, Dubois B, Holloway R, Jankovic J, Kulisevsky J, Lang AE, Lees A, Leurgans S, LeWitt PA, Nyenhuis D, Olanow CW, Rascol O, Schrag A, Teresi JA, van Hilten JJ, LaPelle N, Movement Disorder Society URTF (2008) Movement Disorder Society-sponsored revision of the Unified Parkinson's Disease Rating Scale (MDS-UPDRS): scale presentation and clinimetric testing results. Mov Disord 23(15):2129-2170. https://doi.org/10.1002/mds.22340

16. Hoehn MM, Yahr MD (2001) Parkinsonism: onset, progression, and mortality. 1967. Neurology 57(10 Suppl 3):S11-S26

17. Lawson RA, Yarnall AJ, Duncan GW, Khoo TK, Breen DP, Barker RA, Collerton D, Taylor JP, Burn DJ (2014) Severity of mild cognitive impairment in early Parkinson's disease contributes to poorer quality of life. Parkinsonism Relat Disord 20(10):10711075. https://doi.org/10.1016/j.parkreldis.2014.07.004

18. Mak E, Su L, Williams GB, Firbank MJ, Lawson RA, Yarnall AJ, Duncan GW, Owen AM, Khoo TK, Brooks DJ, Rowe JB, Barker RA, Burn DJ, O'Brien JT (2015) Baseline and 
longitudinal grey matter changes in newly diagnosed Parkinson's disease: ICICLE-PD study. Brain 138(Pt 10):2974-2986. https://doi.org/10.1093/brain/awv211

19. Stebbins GT, Goetz CG, Burn DJ, Jankovic J, Khoo TK, Tilley BC (2013) How to identify tremor dominant and postural instability/gait difficulty groups with the movement disorder society unified Parkinson's disease rating scale: comparison with the unified Parkinson's disease rating scale. Mov Disord 28(5):668670. https://doi.org/10.1002/mds. 25383

20. Tomlinson CL, Stowe R, Patel S, Rick C, Gray R, Clarke CE (2010) Systematic review of levodopa dose equivalency reporting in Parkinson's disease. Mov Disord 25(15):2649-2653. https ://doi.org/10.1002/mds.23429

21. Litvan I, Goldman JG, Troster AI, Schmand BA, Weintraub D, Petersen RC, Mollenhauer B, Adler CH, Marder K, WilliamsGray CH, Aarsland D, Kulisevsky J, Rodriguez-Oroz MC, Burn DJ, Barker RA, Emre M (2012) Diagnostic criteria for mild cognitive impairment in Parkinson's disease: Movement Disorder Society Task Force guidelines. Mov Disord 27(3):349-356. https://doi.org/10.1002/mds.24893

22. Lawson RA, Yarnall AJ, Duncan GW, Breen DP, Khoo TK, Williams-Gray CH, Barker RA, Burn DJ, group I-Ps (2017) Stability of mild cognitive impairment in newly diagnosed Parkinson's disease. J Neurol Neurosurg Psychiatry 88(8):648-652. https://doi.org/10.1136/jnnp-2016-315099

23. Goldman JG, Holden S, Bernard B, Ouyang B, Goetz CG, Stebbins GT (2013) Defining optimal cutoff scores for cognitive impairment using Movement Disorder Society Task Force criteria for mild cognitive impairment in Parkinson's disease. Mov Disord 28(14):1972-1979. https://doi.org/10.1002/mds.25655

24. Pereira JB, Svenningsson P, Weintraub D, Bronnick K, Lebedev A, Westman E, Aarsland D (2014) Initial cognitive decline is associated with cortical thinning in early Parkinson disease. Neurology 82(22):2017-2025. https://doi.org/10.1212/ WNL.0000000000000483

25. Smith SM, Jenkinson M, Johansen-Berg H, Rueckert D, Nichols TE, Mackay CE, Watkins KE, Ciccarelli O, Cader MZ, Matthews PM, Behrens TE (2006) Tract-based spatial statistics: voxelwise analysis of multi-subject diffusion data. Neuroimage 31(4):1487-1505. https://doi.org/10.1016/j.neuroimage 2006.02 .024

26. Smith SM, Jenkinson M, Woolrich MW, Beckmann CF, Behrens TE, Johansen-Berg H, Bannister PR, De Luca M, Drobnjak I, Flitney DE, Niazy RK, Saunders J, Vickers J, Zhang Y, De Stefano N, Brady JM, Matthews PM (2004) Advances in functional and structural MR image analysis and implementation as FSL. Neuroimage 23(Suppl 1):S208-S219. https://doi.org/10.1016/j. neuroimage.2004.07.051

27. Winkler AM, Ridgway GR, Webster MA, Smith SM, Nichols TE (2014) Permutation inference for the general linear model. Neuroimage 92:381-397. https://doi.org/10.1016/j.neuroimage 2014.01 .060

28. Firbank MJ, Minett T, O'Brien JT (2003) Changes in DWI and MRS associated with white matter hyperintensities in elderly subjects. Neurology 61(7):950-954

29. Simons JS, Spiers HJ (2003) Prefrontal and medial temporal lobe interactions in long-term memory. Nat Rev Neurosci 4(8):637648. https://doi.org/10.1038/nrn1178

30. Alexander GE, Crutcher MD (1990) Functional architecture of basal ganglia circuits: neural substrates of parallel processing. Trends Neurosci 13(7):266-271

31. Bonelli RM, Cummings JL (2007) Frontal-subcortical circuitry and behavior. Dialogues Clin Neurosci 9(2):141-151

32. Ye Z, Altena E, Nombela C, Housden CR, Maxwell H, Rittman T, Huddleston C, Rae CL, Regenthal R, Sahakian BJ, Barker RA, Robbins TW, Rowe JB (2015) Improving response inhibition in
Parkinson's disease with atomoxetine. Biol Psychiatry 77(8):740 748. https://doi.org/10.1016/j.biopsych.2014.01.024

33. Atkinson-Clement C, Pinto S, Eusebio A, Coulon O (2017) Diffusion tensor imaging in Parkinson's disease: review and metaanalysis. Neuroimage Clin 16:98-110. https://doi.org/10.1016/j. nicl.2017.07.011

34. Ofori E, Pasternak O, Planetta PJ, Li H, Burciu RG, Snyder AF, Lai S, Okun MS, Vaillancourt DE (2015) Longitudinal changes in free-water within the substantia nigra of Parkinson's disease. Brain 138(Pt 8):2322-2331. https://doi.org/10.1093/brain/awv13 6

35. Modrego PJ, Fayed N, Artal J, Olmos S (2011) Correlation of findings in advanced MRI techniques with global severity scales in patients with Parkinson disease. Acad Radiol 18(2):235-241. https://doi.org/10.1016/j.acra.2010.09.022

36. Zhan W, Kang GA, Glass GA, Zhang Y, Shirley C, Millin R, Possin KL, Nezamzadeh M, Weiner MW, Marks WJ Jr, Schuff N (2012) Regional alterations of brain microstructure in Parkinson's disease using diffusion tensor imaging. Mov Disord 27(1):90-97. https://doi.org/10.1002/mds.23917

37. Nigro S, Riccelli R, Passamonti L, Arabia G, Morelli M, Nistico R, Novellino F, Salsone M, Barbagallo G, Quattrone A (2016) Characterizing structural neural networks in de novo Parkinson disease patients using diffusion tensor imaging. Hum Brain Mapp 37(12):4500-4510. https://doi.org/10.1002/hbm.23324

38. Lo RY, Tanner CM, Albers KB, Leimpeter AD, Fross RD, Bernstein AL, McGuire V, Quesenberry CP, Nelson LM, Van Den Eeden SK (2009) Clinical features in early Parkinson disease and survival. Arch Neurol 66(11):1353-1358. https://doi.org/10.1001/ archneurol.2009.221

39. Vervoort G, Leunissen I, Firbank M, Heremans E, Nackaerts E, Vandenberghe W, Nieuwboer A (2016) Structural brain alterations in motor subtypes of Parkinson's Disease: evidence from probabilistic tractography and shape analysis. PLoS One 11(6):e0157743. https://doi.org/10.1371/journal.pone.0157743

40. Hall JM, Ehgoetz Martens KA, Walton CC, O'Callaghan C, Keller PE, Lewis SJ, Moustafa AA (2016) Diffusion alterations associated with Parkinson's disease symptomatology: A review of the literature. Parkinsonism Relat Disord 33:12-26. https://doi. org/10.1016/j.parkreldis.2016.09.026

41. Schwarz CG, Reid RI, Gunter JL, Senjem ML, Przybelski SA, Zuk SM, Whitwell JL, Vemuri P, Josephs KA, Kantarci K, Thompson PM, Petersen RC, Jack CR Jr., Alzheimer's Disease Neuroimaging I (2014) Improved DTI registration allows voxel-based analysis that outperforms tract-based spatial statistics. Neuroimage 94:6578. https://doi.org/10.1016/j.neuroimage.2014.03.026

42. Melzer TR, Myall DJ, MacAskill MR, Pitcher TL, Livingston L, Watts R, Keenan RJ, Dalrymple-Alford JC, Anderson TJ (2015) Tracking Parkinson's disease over one year with multimodal magnetic resonance imaging in a group of older patients with moderate disease. PLoS One 10(12):e0143923. https://doi.org/10.1371/ journal.pone. 0143923

43. Rossi ME, Ruottinen H, Saunamaki T, Elovaara I, Dastidar P (2014) Imaging brain iron and diffusion patterns: a follow-up study of Parkinson's disease in the initial stages. Acad Radiol 21(1):64-71. https://doi.org/10.1016/j.acra.2013.09.018

44. Fernando MS, O'Brien JT, Perry RH, English P, Forster G, McMeekin W, Slade JY, Golkhar A, Matthews FE, Barber R, Kalaria RN, Ince PG (2004) Comparison of the pathology of cerebral white matter with post-mortem magnetic resonance imaging (MRI) in the elderly brain. Neuropathol Appl Neurobiol 30(4):385-395. https://doi.org/10.1111/j.1365-2990.2004.00550 .X

45. Fernando MS, Ince PG (2004) Vascular pathologies and cognition in a population-based cohort of elderly people. J Neurol Sci 226(1-2):13-17. https://doi.org/10.1016/j.jns.2004.09.004 
46. Melzer TR (2013) The evolution of diffusion tensor imaging in Parkinson's disease research. Mov Disord 28(9):1316. https://doi. org/10.1002/mds.25566

47. Alexander AL, Lee JE, Lazar M, Field AS (2007) Diffusion tensor imaging of the brain. Neurotherapeutics 4(3):316-329. https://doi. org/10.1016/j.nurt.2007.05.011

48. Jankovic J, Kapadia AS (2001) Functional decline in Parkinson disease. Arch Neurol 58(10):1611-1615
49. Rowe JB, Hughes L, Ghosh BC, Eckstein D, Williams-Gray CH, Fallon S, Barker RA, Owen AM (2008) Parkinson's disease and dopaminergic therapy-differential effects on movement, reward and cognition. Brain 131(Pt 8):2094-2105. https://doi. org/10.1093/brain/awn112

\section{Affiliations}

Thais Minett ${ }^{1,2} \cdot \mathrm{Li} \mathrm{Su}^{3,17} \cdot$ Elijah Mak $^{3} \cdot$ Guy Williams $^{4} \cdot$ Michael Firbank $^{5} \cdot$ Rachael A. Lawson $^{5} \cdot$ Alison J. Yarnall $^{5}$. Gordon W. Duncan ${ }^{6}$. Adrian M. Owen ${ }^{7,8}$. Tien K. Khoo ${ }^{9}$. David J. Brooks ${ }^{5,10,11}$. James B. Rowe ${ }^{12,13,14}$. Roger A. Barker ${ }^{15}$. David Burn ${ }^{16}$. John T. O'Brien ${ }^{3}$

1 Department of Radiology, University of Cambridge, Cambridge, UK

2 Cambridge Institute of Public Health, University of Cambridge, Forvie Site, Cambridge Biomedical Campus, Cambridge CB2 OSR, UK

3 Department of Psychiatry, University of Cambridge, Cambridge, UK

4 Wolfson Brain Imaging Centre, University of Cambridge, Cambridge, UK

5 Institute of Neuroscience, Newcastle University, Newcastle upon Tyne, UK

6 Centre for Clinical Brain Sciences, University of Edinburgh, Edinburgh, UK

7 Brain and Mind Institute, University of Western Ontario, London, Canada

8 Department of Psychology, University of Western Ontario, London, Canada

9 School of Medicine, University of Wollongong, Wollongong, NSW, Australia
10 Division of Neuroscience, Imperial College London, London, UK

11 Institute of Clinical Medicine, Aarhus University, Aarhus, Denmark

12 Department of Clinical Neurosciences, University of Cambridge, Cambridge, UK

13 Medical Research Council, Cognition and Brain Sciences Unit, Cambridge, UK

14 Behavioural and Clinical Neuroscience Institute, University of Cambridge, Cambridge, UK

15 John van Geest Centre for Brain Repair, University of Cambridge, Cambridge, UK

16 Faculty of Medical Sciences, Newcastle University, Newcastle upon Tyne, UK

17 China-UK Centre for Cognition and Ageing Research, Southwest University, Chongqing, China 